\title{
Assessment of Linear Discrimination and Nonlinear Discrimination Analysis in Diagnosis Alzheimer's Disease in Early Stages
}

\author{
Roya Golestani ${ }^{1}$, Akbar Gharbali ${ }^{2}$, Surena Nazarbaghi ${ }^{3}$ \\ ${ }^{1}$ Medical Physics Department, Urmia Medical Sciences University, Urmia, Iran \\ ${ }^{2}$ Department of Medical Physics, Faculty of Medicine, Urmia Medical Sciences University, Urmia, Iran \\ ${ }^{3}$ Department of Neurology, Emam Hospital, Urmia Medical Sciences University, Urmia, Iran \\ Email: Re.golestani28@gmail.com, ^gharbali@yahoo.com,nazarbaghi_s@umsu.ac.ir
}

How to cite this paper: Golestani, R., Gharbali, A. and Nazarbaghi, S. (2020) Assessment of Linear Discrimination and Nonlinear Discrimination Analysis in Diagnosis Alzheimer's Disease in Early Stages. Advances in Alzheimer's Disease, 9, 21-32.

https://doi.org/10.4236/aad.2020.92002

Received: May 14, 2020

Accepted: June 25, 2020

Published: June 28, 2020

Copyright $\odot 2020$ by author(s) and Scientific Research Publishing Inc. This work is licensed under the Creative Commons Attribution International License (CC BY 4.0).

http://creativecommons.org/licenses/by/4.0/

\begin{abstract}
Introduction: The purpose of this study is to evaluate discriminating power of two texture analysis, linear discriminant analysis and nonlinear discriminant analysis, in classifying atrophy of Alzheimer's disease and atrophy of aging. Methods: The database included 24 regions of interest of Alzheimer patients and 24 regions of interest of aging people in hippocampus region. Linear discriminant analysis and nonlinear discriminant analysis were used for texture analysis. The first nearest neighbor classifier was applied to features resulting from linear discriminant analysis. Nonlinear discriminant analysis features were classified by using an artificial neural network. The confusion matrix and Receiver Operating Characteristic (ROC) curve analysis were used to examine the performance of texture analysis method. Result: Nonlinear discriminant analysis indicates the best performance for classification of atrophy of Alzheimer's disease and atrophy of aging. Conclusion: Our result showed computer aided diagnosis has high potential discriminating power in classifying Alzheimer's disease in early stage.
\end{abstract}

\section{Keywords}

Atrophy, Alzheimer's Disease, Analysis

\section{Introduction}

Alzheimer's disease is a brain disorder characterized by a progressive dementia that occurs in middle or late life. The pathologic characteristics are degeneration of specific nerve cells, presence of neurotic plaques, and neurofibrillary tangles. 
A Work Group on the Diagnosis of Alzheimer's Disease was established by the National Institute of Neurological and Communicative Disorders and Stroke (NINCDS) and the Alzheimer's Disease and Related Disorders Association (ADRDA). The group intended to establish and to describe clinical criteria for the diagnosis of Alzheimer's disease of particular importance for research protocols and to describe approaches that would be useful for assessing the natural history of the disease [1].

Alzheimer's disease (AD) is the most common form of dementia which is a degenerative disorder of the brain that leads to memory loss [2].

The only method of definitively diagnosing $\mathrm{AD}$ is a brain autopsy [3]. Functional imaging allows the physician to determine how effectively the brain cells are working. A functional MRI or positron emission tomography (PET) scan can be used [4].

Mini Mental State Examination (MMSE) and Clinical Dementia Rating (CDR) are two of the most commonly used tests in the assessment of AD [5] [6]. MMSE consists of a series of clinical and psychometric assessment through ability of solving problems. The maximum MMSE score is 30 points. A score less than 12 indicates severe dementia, 13 to 20 recommends moderate dementia, 20 to 24 suggests mild dementia and 24 to 30 represents Normal Controls (NC). In parallel, CDR is applied to evaluate memory, orientation, judgment, and problem solving, home and hobbies and personal care. A score of 0 , represents normal controls, 0.5 , very mild dementia, 1 , mild dementia, 2 , moderate dementia and 3 , severe dementia [7].

The revised criteria for the diagnosis of $\mathrm{AD}$ were proposed in 2007 by the National Institute of Neurological Disorders and Stroke-Alzheimer Disease and Related Disorders working group. According to and due to the uncertainty of clinical diagnosis, the clinical assessment should include at least one supportive feature: Medial Temporal Lobe (MTL) atrophy as seen in structural Magnetic Structural imaging scans, including magnetic resonance imaging (MRI) and computed tomography (CT), provides information about the shape and volume of the brain [8].

Computer aid diagnosis (CAD) can be useful tool for evaluating texture figures. This method is cost-efficiency and non-invasive. It boosts the accuracy of diagnosing many diseases including Alzheimer's disease. One of the main pathological features of $\mathrm{AD}$ is neuronal lost with consequence that brain atrophy is observable in MR images [9] [10]. However, the atrophy due to AD in early stage is difficult to distinguish from that caused by normal aging [11].

Several studies assess the diagnosis Alzheimer's disease with other groups such as aging group or Mild Cognition Impairment (MCI) patients by using different methods such as Voxel-based Morphometric (VBM), volumetric measurements in specific Region of Interest (ROIs), cortical thickness measurements, shape analysis and texture analysis. VBM (voxel based morphometric) describes global changes or atrophy of deep cerebral structures [7]. E vans et al. 
revealed a mean standard $0.5 \%$ per year in NC (normal control) based on VBM [12]. Karas et al. used VBM, demonstrating global reduced GM (gray matter) volume includes the cerebellum, medial thalamus and head of the caudate nucleus as well of the Cingulum in AD patients [13]. Another study by Karas et al. an analyzed the patterns of GM loss in order to examine what characterizes MCI and what determines the differences with AD by using VBM method [14]. Colliot and Chupin et al. used hippocampal volume to distinguish NC from MCI and $\mathrm{AD}$ patients [15]. The results of their study revealed significant hippocampal volume reductions in all groups of patients. Specifically, there was a $32 \%$ volume reduction between $\mathrm{AD}$ and $\mathrm{NC}$, a 19\% reduction between $\mathrm{MCI}$ and $\mathrm{NC}$ and finally, a 15\% reduction between AD and MCI [16]. According to Pennanen the hippocampal atrophy in $\mathrm{AD}$ patients were more pronounced than MCI patients [17]. Several studies used hippocampal volumetric measurements and confirmed that hippocampal atrophy, can constitute a useful diagnostic biomarker [7] [18].

Several studies performed based on texture analysis such as, Freeborough and Fox (1996-1998) analyzed whole brain of 24 AD patients and 40 normal groups by using texture analysis. Simoes et al. (2012) performed similar study for MCI patients. Jing Zhang (2012) evaluated accuracy for diagnosis AD by using texture analysis [3] [9] [19] [20].

Texture analysis detects pathological changes that cannot be perceived by the human eyes using conventional MRI techniques. Texture features are analyzed coming from six main categories in the proposed computer aided diagnosis (CAD) system including: Histogram (statistical class), Absolute gradient (statistical class), Run length matrix (statistical class), Co-occurrence matrix (statistical class), Auto-Regressive (AR) model (model class) and Wavelets (transform class) [21]. The present study used TA to evaluate texture features extracted from MR images to differentiate between atrophy of Alzheimer and atrophy of normal aging.

\section{Materials and Methods}

The present study used texture analysis by using MaZda software (version 4 - 6) to evaluate texture features extracted from MR images.

In this study, 12 patients digital MRI images, aged $64.4 \pm 9.43$ (mean age \pm standard deviation) with a clinically definite Alzheimer's disease and 12 healthy elderly images ( 9 male and 4 females) aged $68.54 \pm 7.02$, were collected during 6 months and relevant neurologist approved them. $\mathrm{T}_{2} \mathrm{~W}$-TSE MR images of patient and healthy subjects were acquired from a $1.5 \mathrm{~T}$ scanner (Philips Achieva, Philips Medical system, Best, the Netherlands). Matrix $256 \times 126, \mathrm{TR}=15, \mathrm{TE}=$ $5, \mathrm{TI}=0, \mathrm{FA}=20$, Thickness $=10$, Spacing $=20$. Alzheimer lesion in MRI images were identified and placed with the help of expert neurologist and confirmed by a radiologist. Criteria were used to select region of interest (ROI) include two ROIs were selected for each subject, ROI placed in right and left of hippocampus region and all ROIs has the same size and chooses in the same 
place of hippocampus. One MR image was input in MaZda (version 4.6, technical university of Lodz, Institute of electronics) for texture analysis. Three normalizations were applied for each ROI. The first, $\mathrm{N}_{1}$, was the default and no normalization has applied. The second, $\mathrm{N}_{2}$, was 3-sigma, in which the ROI intensities have limited in the range $(\mu-3 \sigma, \mu+3 \sigma)$ where $\mu$ and $\sigma$, respectively, where the mean value and standard deviation of the intensity. The third normalization was $\mathrm{N}_{3}, 1 \%-99 \%$, in which the image intensity ranges were normalized between darkness level (accumulated histogram is equal to $1 \%$ of total) and the brightness level (accumulate histogram $=99 \%$ of total) inside the ROI [22]. Over 270 texture features extracted based on Histogram, Absolute gradient (spatial variation of grey level values, Run-length matrix (counts of pixel runs with the specified gray-scale value and length in a given direction), Co-occurrence matrix (information about the distribution of pairs of pixels separated by given distance and direction), Auto regressive model (description of correlation between neighboring pixels) and Wavelet decomposition image frequency and different scales). Not all 270 texture features were suitable or effective for use differentiating atrophy of Alzheimer and atrophy of normal aging. Two algorithms of feature reduction (Fisher) and lowest probability of classification error and average correlation coefficients (POE + ACC) were employed to reduce the parameters to the best 10 texture features. These features were analyzed using both standard and nonstandard states. Linear discriminant analysis (LDA) and nonlinear discriminant analysis (NDA) were used to analyze data. First nearest neighbor (1-NN) classifier has used for the features from LDA and artificial neural network (ANN) used for NDA analysis. ROC curve was employed for comparing the maximum discrimination among these analyses and area under curve (AUC) was calculated for each one. In addition two factors include sensitivity (SN) specificity (SP) are applied to assess performance of these methods. Sensitivity is the ability of test to correctly identify those with the disease (true positive rate), whereas test specificity is the ability of the test to correctly identify those without the disease (true negative rate).

\section{Results}

Total 48 cases (24 patients, 24 elderly) were selected to evaluate the classification and accuracy of proposed method. Parameters were extracted from fisher algorithm in default state (without normalization) include Co-Occurrence Matrix (7parameters), Gradients (3 parameters), parameters in POE + ACC include 5 Co-Occurrence matrix, 2 AR model, 1Run-Length matrix, 1 gradient and 1 wavelet. In 3 sigma normalization, parameters from fisher algorithm were Co-Occurrence Matrix ( 9 parameters), Gradient (1 parameter) parameters in POE + ACC algorithm include 5 Co-Occurrence Matrix, 2 Wavelet, 2 absolute gradients and 1 histogram. In 1\% - 99\% normalization parameters include 8 Co-Occurrence Matrix and 1 absolute gradient and 1 Run-Length matrix, parameters extracted by POE + ACC algorithm include 8 Co-Occurrence matrix, 1 histogram and 1 Wavelet (Tables 1-3). 
Table 1. The best ten Fisher and POE + ACC features in default state.

\begin{tabular}{ccccc}
\hline ROI $^{1}$ & \multicolumn{3}{c}{ Texture Features parameters selection algorithms } \\
\hline Normalization & \multicolumn{2}{c}{ Fisher } & \multicolumn{2}{c}{ POE + ACC } \\
\hline & Feature Name & Value & Feature Name & Value \\
\hline & GrKurtosis & 0.4355 & Teta2 & 0.4209 \\
& GrSkewness & 0.4194 & S[0,4]Correlat & 0.4304 \\
& GrVariance & 0.3712 & S[0,1]Correlat & 0.4612 \\
& S[0,3]DifEntrp & 0.3221 & 45dgrShrtREmp & 0.4662 \\
Default & S[2,2]Correlat & 0.2985 & S[4,0]Correlat & 0.4754 \\
& S[0,4]Correlat & 0.2820 & S[2,0]SumAverg & 0.4824 \\
& S[0,1]SumAverg & 0.2807 & S[0,2]Correlat & 0.4867 \\
& S[1,1]SumAverg & 0.2757 & Teta1 & 0.4903 \\
& S[1,0]SumAverg & 0.2731 & GrKurtosis & 0.4904 \\
& S[2,0]SumAverg & 0.2720 & WavEnLL-S-1 & 0.7500 \\
\hline
\end{tabular}

Table 2. The best ten Fisher and POE + ACC features in 3 Sigma state.

\begin{tabular}{ccccc}
\hline \multicolumn{3}{c}{ Texture Features parameters selection algorithms } \\
\hline \multirow{2}{*}{ ROI $^{1}$ Normalization } & Fisher & \multicolumn{2}{c}{ POE + ACC ${ }^{2}$} \\
\hline & Feature Name & Value & Feature Name & Value \\
\hline & S[0,1]SumDifSqs & 0.6971 & S[0,1]DifVarnc & 0.4189 \\
S[0,3]DifVarnc & 0.6873 & S[3,0]Correlat & 0.4433 \\
& S[1,0]SumAverg & 0.6166 & Perc50\% & 0.4506 \\
S[4,0]SumAverg & 0.5837 & WavEnLL-s-1 & 0.4593 \\
3 Sigma & S[1,1]SumDfSqs & 0.4840 & S[0,2]InvDfMom & 0.4619 \\
& Kurtosis & 0.4773 & GrKurtosis & 0.4747 \\
& S[0,2]InvDfMom & 0.3789 & WavEnHL-S-1 & 0.4749 \\
& S[3,0]Correlat & 0.3699 & Skewness & 0.4868 \\
S[0,2]SumAverg & 0.3678 & S[2,2]SumDfSqs & 0.4880 \\
& S[1,-1]SumDfSq & 0.3625 & S[1,0]SumDfSqs & 0.7917 \\
\hline
\end{tabular}

Table 3. The best ten Fisher and POE + ACC features in 1\% - 99\% state.

\begin{tabular}{ccccc}
\hline \multicolumn{4}{c}{ Texture Features parameters selection algorithms } \\
\hline ROI $^{1}$ Normalization & \multicolumn{3}{c}{ Fisher } & \multicolumn{2}{c}{ POE + ACC } \\
& Feature Name & Value & Feature Name & Value \\
\hline & S[0,3]DifVarnc & 0.6931 & Perc50\% & 0.4260 \\
& S[3,0]DifVarnc & 0.5730 & S[1,-1]InvDfMo & 0.4423 \\
Kurtosis & 0.4773 & S[0,1]SumVarnc & 0.4581 \\
& S(3,0)Contrast & 0.4704 & S[4,0]Contrast & 0.4812 \\
& S(0,1)SumVarrnc & 0.3818 & S[0,2]InvDfMom & 0.4831 \\
& 135dr-GLevNon & 0.3722 & S[0,3]DifVarnc & 0.4857 \\
& S[3,0]InvDfMom & 0.3622 & S[2,2]SumAverge & 0.4898 \\
& S[3,0]Correlat & 0.3572 & S[2,0]InvDfMom & 0.4933 \\
& S[0,1]SumofSqs & 0.3318 & S[3,0]Correlat & 0.4937 \\
& S[0,2]InvDfMom & 0.3309 & WavEnLL-s- 1 & 0.7708 \\
\hline
\end{tabular}

Analyzing of parameters considered in two standard and nonstandard states. Standardization of parameters calculated by:

${ }^{1}$ Region of interest.

${ }^{2}$ Correlation Probability of classification error and average coefficient. 


$$
X_{i}^{\prime}=\frac{X_{i}-\mu}{\sigma}
$$

In this formula, $X_{i}$ and $X_{i}^{\prime}$ are parameters before and after standardization. $\mu$ is the mean value and $\sigma$ is the standard deviation. The results of LDA analysis in three states of default state, 3 sigma, 1\% - 99\% listed in Table 4. According to Table 4, 1\% - 99\% normalization had the highest effect on results and standardization had no effect on results.

The result of NDA analysis in three states, without normalization, 3 sigma, 1\% - 99\% normalization listed in Table 5. In general, normalization raised the operation of NDA analysis. Standardization has no effect on NDA analysis except standardization reduces sensitivity in default mode and $1 \%-99 \%$ normalization when using the POE + ACC algorithm.

ROC curves with the highest value of two analyses, LDA and NDA have been shown in Figures 1-3. The area under curves has been also calculated. The highest value of area under curve (AUC) belonged to NDA analysis.

\section{Discussion}

The primary objective to this study was to differentiate between atrophy of Alzheimer disease and atrophy of aging using MR imaging. 48 ROIs were evaluated. For each individual, two region of interest (ROI) was chosen in hippocampus region of coronal image (Figure 4).

Table 4. Differentiation performance results of the Alzheimer from normal aging via LDA ${ }^{3}$.

\begin{tabular}{|c|c|c|c|c|c|}
\hline \multicolumn{2}{|c|}{ ROC analysis } & \multirow[t]{2}{*}{ standardization } & \multirow[t]{2}{*}{ Feature reduction } & \multirow{2}{*}{$\begin{array}{c}\text { Normalization } \\
\text { schemes }\end{array}$} & \multirow{2}{*}{$\begin{array}{c}\text { Texture } \\
\text { analysis Methods }\end{array}$} \\
\hline $\mathrm{SP}^{4}(\%)$ & $\mathrm{SN}^{4}(\%)$ & & & & \\
\hline 0.83 & 0.83 & $\mathrm{~S}^{5}$ & \multirow{2}{*}{ Fisher } & \multirow{4}{*}{ Default } & \multirow{11}{*}{$\begin{array}{l}\text { LDA with K-NN } \\
\text { classifier }\end{array}$} \\
\hline 0.83 & 0.83 & $\mathrm{NS}^{6}$ & & & \\
\hline 0.77 & 0.73 & $S$ & \multirow{2}{*}{$\mathrm{POE}+\mathrm{ACC}$} & & \\
\hline 0.77 & 0.73 & NS & & & \\
\hline 0.86 & 0.75 & $S$ & \multirow[t]{2}{*}{ Fisher } & \multirow{3}{*}{3 Sigma } & \\
\hline $\begin{array}{l}0.86 \\
0.68\end{array}$ & $\begin{array}{c}0.75 \\
0.705\end{array}$ & NS & & & \\
\hline 0.68 & 0.705 & NS & $\mathrm{POE}+\mathrm{ACC}$ & & \\
\hline 0.83 & 0.88 & $\mathrm{~S}$ & \multirow{2}{*}{ Fisher } & \multirow{4}{*}{$1 \%-99 \%$} & \\
\hline 0.83 & 0.88 & NS & & & \\
\hline 0.705 & 0.75 & $\mathrm{~S}$ & \multirow[t]{2}{*}{$\mathrm{POE}+\mathrm{ACC}$} & & \\
\hline 0.705 & 0.75 & NS & & & \\
\hline
\end{tabular}

${ }^{3}$ Linear Discriminant analysis.

${ }^{4}$ Specificity.

${ }^{5}$ Standard.

${ }^{6}$ Non-standard.

${ }^{7}$ Nearest neighbor classifier. 
Table 5. Differentiation performance results of the Alzheimer from normal aging via $\mathrm{NDA}^{8}$.

\begin{tabular}{|c|c|c|c|c|c|}
\hline \multicolumn{2}{|c|}{ ROC analysis } & \multirow{2}{*}{ standardization } & \multirow{2}{*}{$\begin{array}{l}\text { Feature } \\
\text { reduction }\end{array}$} & \multirow{2}{*}{$\begin{array}{l}\text { Normalization } \\
\text { schemes }\end{array}$} & \multirow{2}{*}{$\begin{array}{c}\text { Texture analysis } \\
\text { Methods }\end{array}$} \\
\hline $\mathrm{SP}^{9}(\%)$ & $\mathrm{SN}^{9}(\%)$ & & & & \\
\hline 0.86 & 0.96 & $S^{10}$ & \multirow{2}{*}{ Fisher } & \multirow{4}{*}{ Default } & \multirow{10}{*}{$\begin{array}{c}\text { NDA with } \text { ANN }^{12} \\
\text { classifier }\end{array}$} \\
\hline 0.86 & 0.96 & $\mathrm{NS}^{11}$ & & & \\
\hline 0.92 & 0.83 & $S$ & & & \\
\hline 0.86 & 0.92 & NS & $\mathrm{POE}+\mathrm{ACC}$ & & \\
\hline 0.83 & 1 & $S$ & Fisher & & \\
\hline $\begin{array}{l}0.83 \\
0.88\end{array}$ & 1 & NS & & 3 Sigma & \\
\hline 0.88 & 0.92 & NS & $\mathrm{POE}+\mathrm{ACC}$ & & \\
\hline 0.86 & 0.96 & $S$ & Fisher & \multirow{3}{*}{$1 \%-99 \%$} & \\
\hline 0.86 & 0.96 & NS & & & \\
\hline $\begin{array}{l}0.88 \\
0.83\end{array}$ & $\begin{array}{l}0.92 \\
0.96\end{array}$ & $\begin{array}{c}\text { S } \\
\text { NS }\end{array}$ & $\mathrm{POE}+\mathrm{ACC}$ & & \\
\hline
\end{tabular}

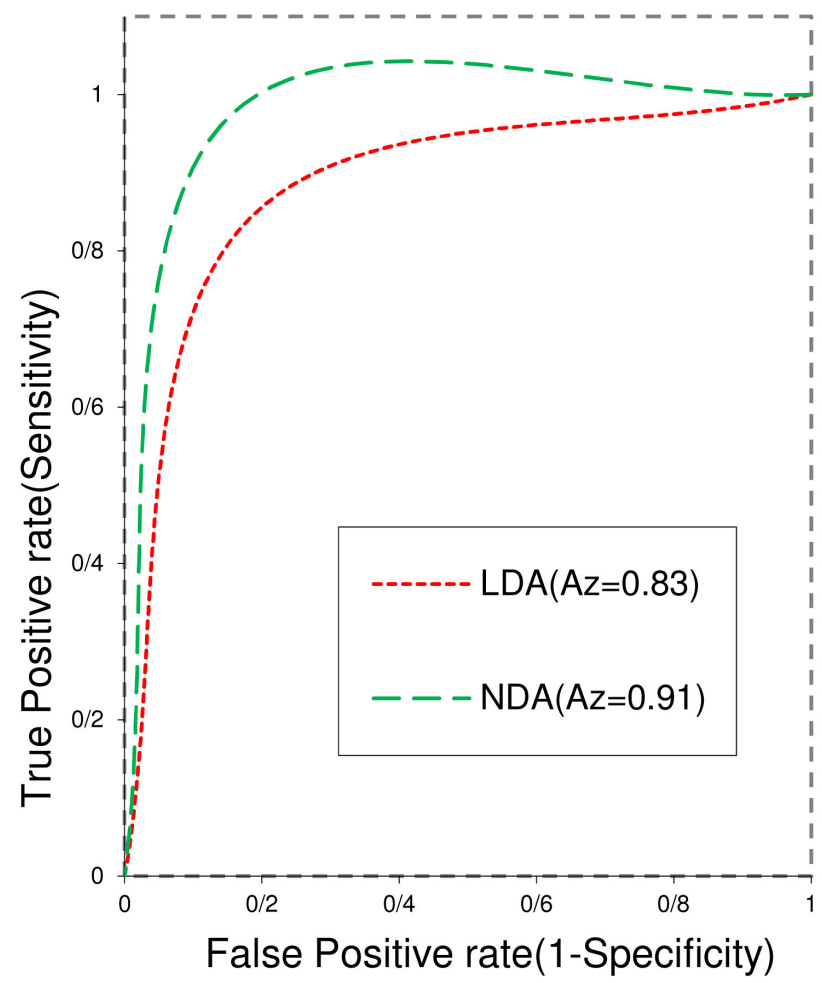

Figure 1. ROC curve with the highest value of LDA and NDA analyses in classification of Alzheimer's disease in early stages and atrophy of normal aging in default state.

\footnotetext{
${ }^{8}$ Nonlinear Discriminant Analysis.

${ }^{9}$ Specificity.

${ }^{10}$ Standard.

${ }^{11}$ Non-standard.

${ }^{12}$ Neural Network Classification.
} 


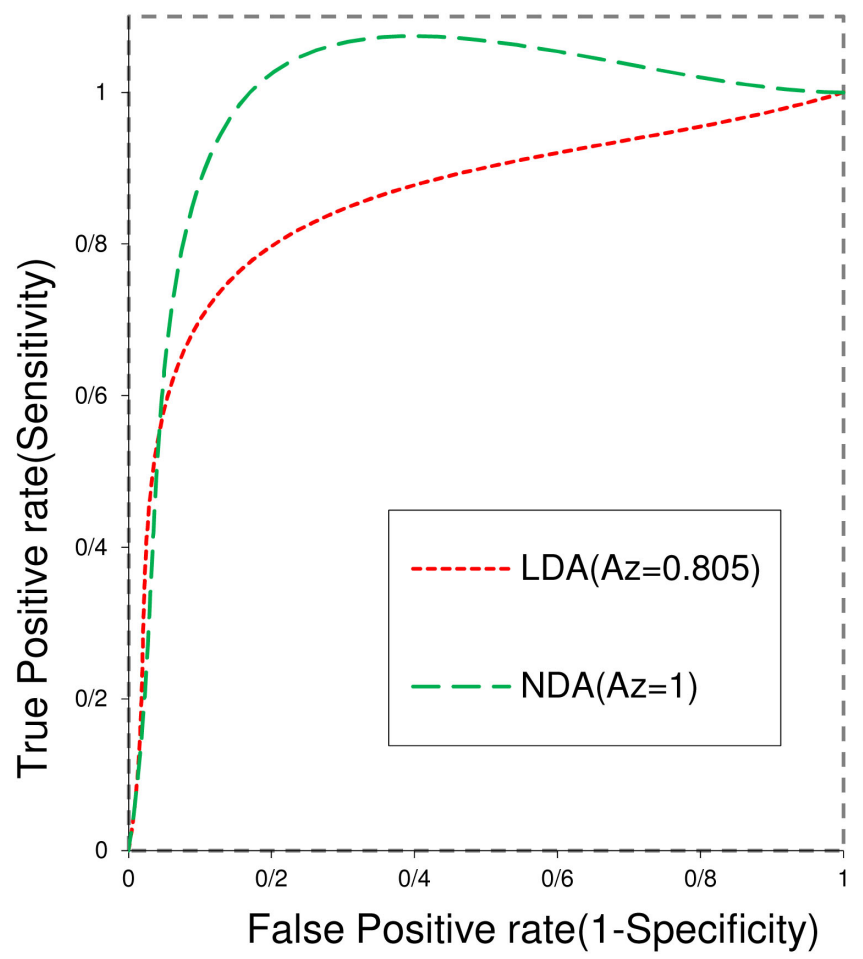

Figure 2. ROC curve with the highest value of LDA and NDA analyses in classification of Alzheimer's disease in early stages and atrophy of normal aging in 3 Sigma state.

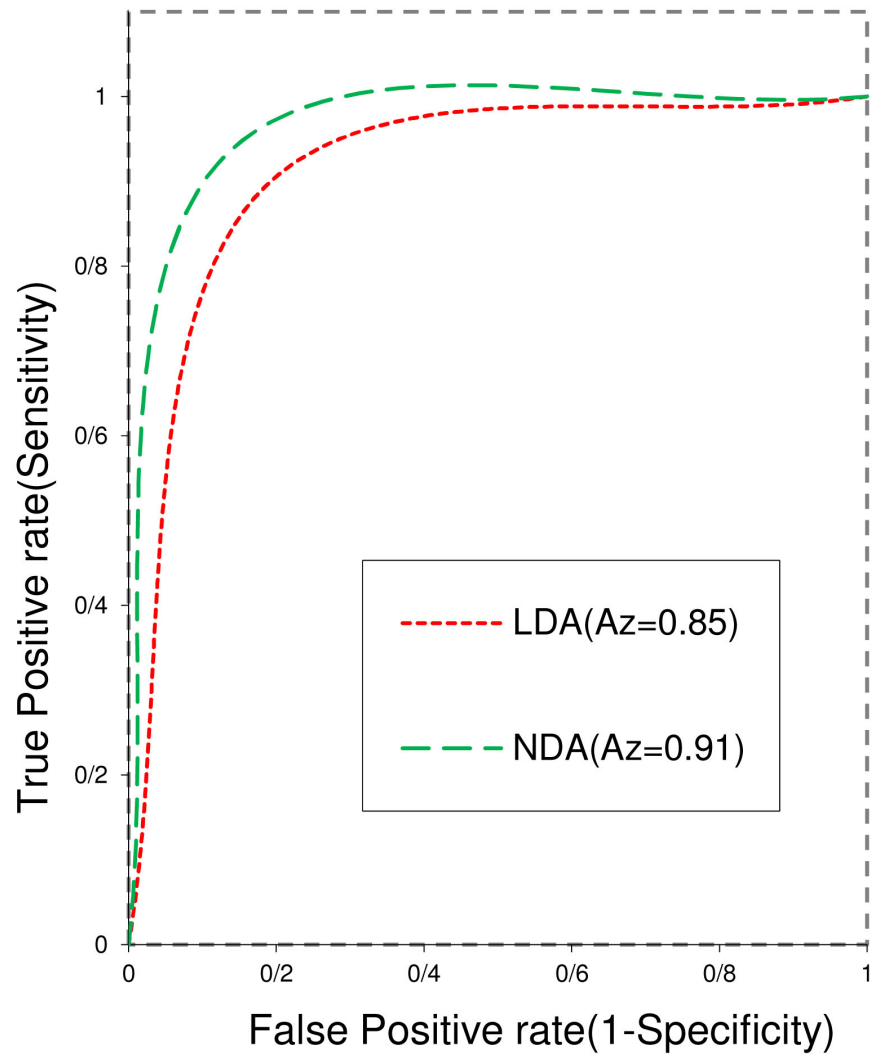

Figure 3. ROC curve with the highest value of LDA and NDA analyses in classification of Alzheimer's disease in early stages and atrophy of normal aging in $1 \%-99 \%$ state. 


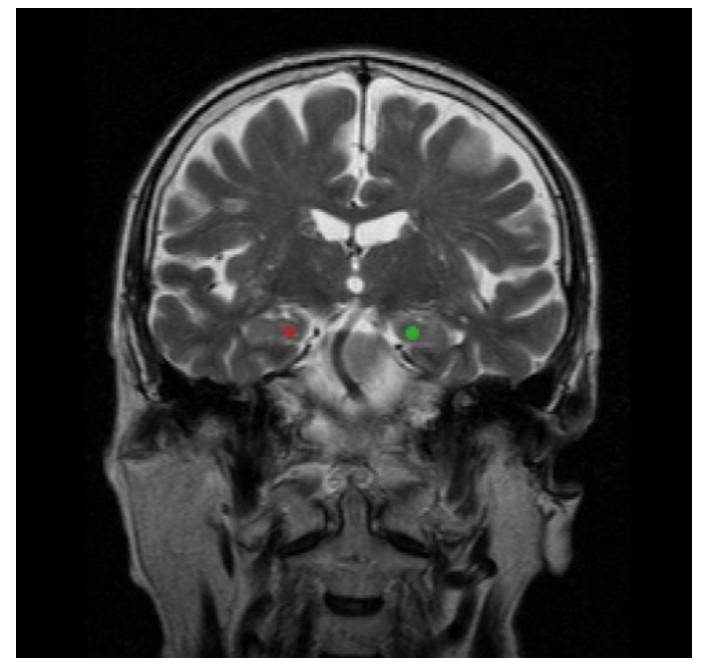

Figure 4. Region of interest (ROI) was chosen in hippocampus region of coronal image.

Two feature reduction methods (Fisher and POE + ACC), two standard and non-standard states and two texture data analysis (LDA, and NDA) were provided per ROI case study. The results show that texture analysis differentiated atrophy of Alzheimer and atrophy of aging with high accuracy. The best parameters extracted from reduction feature algorithms (Fisher and POE + ACC) are generally Co-Occurrence Matrix. In LDA and NDA analyses, standardization has no effect on operation. NDA has the highest area under curve $(A U C=1)$ in 3 Sigma normalization by using fisher algorithm (Figure 5).

In the study by Sørensen et al. the classification capabilities of hippocampal texture were evaluated using Receiver Operating Characteristic (ROC) curves with the corresponding AUC as performance measure. Texture analysis had an AUC of 0.912 in discriminating NC vs $\mathrm{AD}$ and 0.764 between NC vs MCI. For the same groups, the AUC curves for volume analysis were 0.909 and 0.784 respectively [23]. Freeborough and Fox conducted the structural analysis of entire brain in $24 \mathrm{AD}$ patients and 40 people in normal group with $\mathrm{SN}=79 \%, \mathrm{ACC}=$ $91 \%$ and SP $=100 \%$ [9] [19]. Simoes et al. carried out a similar study on MCI patients and the normal group (15 MCI patients and 15 healthy individuals (NC) and the results were as follows: $\mathrm{SN}=85 \%, \mathrm{SP}=95 \%$ and ACC $=87 \%$ [20]. In this study, the highest value of SN and SP was 100 percent for LDA analysis in K-NN classification and NDA analysis in ANN classification. Jing Zhang et al., conducted the 3-dimensional analysis of the structure as a marker for Alzheimer diagnosis. The accuracy of structural analysis based on different ROI is variable from $64.3 \%$ to $964 \%$. Classification accuracy of $1-\mathrm{NN}$ for PCA and LDA analyses is relatively low $(89.7 \%-63.2 \%)$ but the accuracy of ANN classification is relatively high $(98.5 \%-92.6 \%)$ [3]. Present study is based on structure analysis for Alzheimer patients as a useful method in discriminating Alzheimer atrophy from aging atrophy. Structural analysis is able to observer the micro-structural changes in the structure and could be considered an effective method for Alzheimer analysis in early stages. The limitations of this study are as following; the 


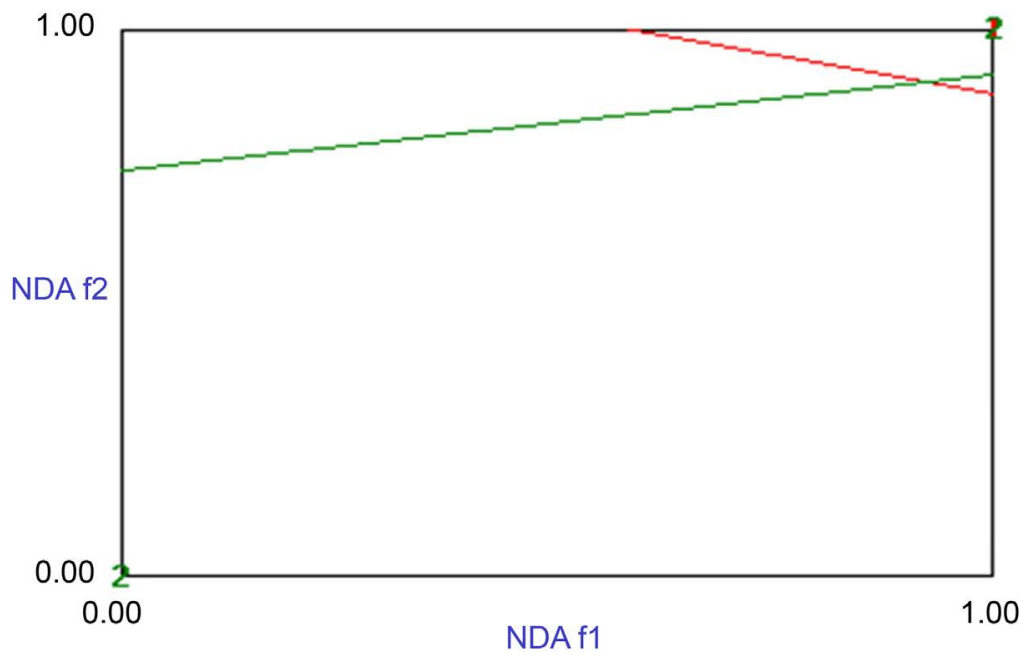

Figure 5. NDA, areas on the nonlinear discriminant analysis (f1 and f2) plane that form a common part of sets belonging to two categories (categoril Alzheimer's disease and category 2 old aging).

number of samples is low and requires more samples to be studied. The method of preparing the images could be different in each patient given the outside factors such as changes in magnetic field and errors related to the shaking or movement of the patient or the system and as a result affect the analyzed images.

\section{Conclusion}

The main advantage of this method is that it can be used as an auxiliary tool to improve accuracy of diagnosis of Alzheimer and requires no additional cost and time. These comparative results showed that the proposed CAD system has the potential to characterize and classify atrophy of Alzheimer's disease and atrophy of aging.

\section{Acknowledgements}

The current study was a part of master thesis which was approved by Urmia University of medical Sciences and designed by Dr Akbar Gharbali (supervisor). All digital images were provided from Imam Khomeini Hospital (Urmia-Iran). We just used digital features of MRI image so that no special criteria of patients included for such study.

I would like to thank Dr Surena Nazarbagh (Neurologist) for his advice and digital MRI images support.

\section{Conflicts of Interest}

The authors declare no conflicts of interest regarding the publication of this paper.

\section{References}

[1] McKhann, G., et al. (1984) Clinical Diagnosis of Alzheimer's Disease Report of the 
NINCDS-ADRDA Work Group under the Auspices of Department of Health and Human Services Task Force on Alzheimer's Disease. Neurology, 34, 939. https://doi.org/10.1212/WNL.34.7.939

[2] Association, A.S. (2010) Alzheimer's Disease Facts and Figures. Alzheimer's \& dementia, 6, 158-194. https://doi.org/10.1016/j.jalz.2010.01.009

[3] Zhang, J., et al. (2012) 3D Texture Analysis on MRI Images of Alzheimer's Disease. Brain Imaging and Behavior, 6, 61-69. https://doi.org/10.1007/s11682-011-9142-3

[4] Emilien, G., et al. (2012) Alzheimer Disease: Neuropsychology and Pharmacology. Birkhäuser, Berlin.

[5] Folstein, M.F., Folstein, S.E. and McHugh, P.R. (1975) "Mini-Mental State": A Practical Method for Grading the Cognitive State of Patients for the Clinician. Journal of Psychiatric Research, 12, 189-198. https://doi.org/10.1016/0022-3956(75)90026-6

[6] Morris, J.C. (1993) The Clinical Dementia Rating (CDR): Current Version and Scoring Rules. Neurology, 43. https://doi.org/10.1212/WNL.43.11.2412-a

[7] Leandrou, S., et al. (2018) Quantitative MRI Brain Studies in Mild Cognitive Impairment and Alzheimer's Disease: A Methodological Review. IEEE Reviews in Biomedical Engineering, 11, 97-111. https://doi.org/10.1109/RBME.2018.2796598

[8] Dubois, B., et al. (2007) Research Criteria for the Diagnosis of Alzheimer's Disease: Revising the NINCDS-ADRDA Criteria. The Lancet Neurology, 6, 734-746. https://doi.org/10.1016/S1474-4422(07)70178-3

[9] Fox, N.C., Freeborough, P.A. and Rossor, M.N. (1996) Visualisation and Quantification of Rates of Atrophy in Alzheimer's Disease. The Lancet, 348, 94-97. https://doi.org/10.1016/S0140-6736(96)05228-2

[10] Scheltens, P. and van de Pol, L. (2012) Atrophy of Medial Temporal Lobes on MRI in "Probable" Alzheimer's Disease and Normal Ageing: Diagnostic Value and Neuropsychological Correlates. Journal of Neurology, Neurosurgery \& Psychiatry, 83, 1038-1040. https://doi.org/10.1136/jnnp-2012-302562

[11] Hwang, E.J., et al. (2016) Texture Analyses of Quantitative Susceptibility Maps to Differentiate Alzheimer's Disease from Cognitive Normal and Mild Cognitive Impairment. Medical Physics, 43, 4718-4728. https://doi.org/10.1118/1.4958959

[12] Evans, M.C., et al. (2010) Volume Changes in Alzheimer's Disease and Mild Cognitive Impairment: Cognitive Associations. European Radiology, 20, 674-682. https://doi.org/10.1007/s00330-009-1581-5

[13] Karas, G., et al. (2003) A Comprehensive Study of Gray Matter Loss in Patients with Alzheimer's Disease Using Optimized Voxel-Based Morphometry. NeuroImage, 18, 895-907. https://doi.org/10.1016/S1053-8119(03)00041-7

[14] Karas, G., et al. (2004) Global and Local Gray Matter Loss in Mild Cognitive Impairment and Alzheimer's Disease. NeuroImage, 23, 708-716. https://doi.org/10.1016/j.neuroimage.2004.07.006

[15] Colliot, O., et al. (2008) Discrimination between Alzheimer Disease, Mild Cognitive Impairment, and Normal Aging by Using Automated Segmentation of the Hippocampus. Radiology, 248, 194-201. https://doi.org/10.1148/radiol.2481070876

[16] Chupin, M., et al. (2007) Anatomically Constrained Region Deformation for the Automated Segmentation of the Hippocampus and the Amygdala: Method and Validation on Controls and Patients with Alzheimer's Disease. NeuroImage, 34, 996-1019. https://doi.org/10.1016/j.neuroimage.2006.10.035

[17] Pennanen, C., et al. (2004) Hippocampus and Entorhinal Cortex in Mild Cognitive Impairment and Early AD. Neurobiology of Aging, 25, 303-310. 
https://doi.org/10.1016/S0197-4580(03)00084-8

[18] Schuff, N., et al. (2009) MRI of Hippocampal Volume Loss in Early Alzheimer's Disease in Relation to ApoE Genotype and Biomarkers. Brain, 132, 1067-1077. https://doi.org/10.1093/brain/awp007

[19] Freeborough, P.A. and Fox, N.C. (1998) MR Image Texture Analysis Applied to the Diagnosis and Tracking of Alzheimer's Disease. IEEE Transactions on Medical Imaging, 17, 475-478. https://doi.org/10.1109/42.712137

[20] Simoes, R., Slump, C. and van Cappellen van Walsum, A.-M. (2012) Using Local Texture Maps of Brain MR Images to Detect Mild Cognitive Impairment. 201221 st International Conference on Pattern Recognition (ICPR), Tsukuba, 11-15 November 2012, 153-156.

[21] Castellano, G., et al. (2004) Texture Analysis of Medical Images. Clinical Radiology, 59, 1061-1069. https://doi.org/10.1016/j.crad.2004.07.008

[22] Szczypiński, P.M., et al. (2009) MaZda-A Software Package for Image Texture Analysis. Computer Methods and Programs in Biomedicine, 94, 66-76.

https://doi.org/10.1016/j.cmpb.2008.08.005

[23] Sørensen, L., et al. (2016) Early Detection of Alzheimer's Disease Using M RI Hippocampal Texture. Human Brain Mapping, 37, 1148-1161.

https://doi.org/10.1002/hbm.23091

\section{Abbreviations}

MRI: Magnetic Resonance Imaging

PCA: Principal Component Analysis

LDA: Linear Discriminant Analysis

NDA: Nonlinear Discriminant Analysis

AD: Alzheimer's disease

MMSE: Mini Mental State Examination

CDR: Clinical Dementia Rating

ROI: Region of Interest

TA: Texture Analysis

ROC: Receiver operating characteristic

CAD: Computer Aim Diagnosis

AUC: Area under Curve

SN: Sensitivity

SP: Specificity

POE + ACC: correlation Probability of classification error and average coefficient

$\mathrm{T}_{2}$ W TSE: $\mathrm{T}_{2}$ Weighted Turbo Spin Echo 